\title{
Effects of Nonlinearity on the Variational Iteration Solutions of Nonlinear Two-Point Boundary Value Problems with Comparison with Respect to Finite Element Analysis
}

\author{
Mehmet Tarık Atay ${ }^{1}$ and Safa Bozkurt Coşkun ${ }^{2}$ \\ ${ }^{1}$ Department of Mathematics, Nigde University, 51245 Nigde, Turkey \\ ${ }^{2}$ Department of Civil Engineering, Nigde University, 51245 Nigde, Turkey \\ Correspondence should be addressed to Safa Bozkurt Coşkun, sbcoskun@nigde.edu.tr
}

Received 12 December 2006; Accepted 10 January 2008

Recommended by Katica Hedrih

Solution of a nonlinear two-point boundary value problem is studied using variational iteration method (VIM) considering its convergence behavior due to the changing nonlinearity effects in the equation. To achieve this, steady Burger equation is first solved by using finite element method (FEM) with a very fine mesh for the comparison of results obtained from VIM. Effect of the nonlinear term in the equation that is multiplied by a constant is taken into account for five different cases by changing the corresponding constant. Results have shown that VIM is a flexible, easy to apply, and promising method for the analysis of nonlinear two-point boundary value problems with the fact that the larger the effect of the nonlinear term of the equation, the slower the convergence rate when compared to FEM solutions.

Copyright ( 2008 M. T. Atay and S. B. Coşkun. This is an open access article distributed under the Creative Commons Attribution License, which permits unrestricted use, distribution, and reproduction in any medium, provided the original work is properly cited.

\section{Introduction}

In the scientific world, numerical solutions of two-point boundary value problems (BVP's) have great importance because of their wide range of applications. These problems have been studied immensely using different numerical approaches such as finite difference method, finite element method, B-spline methods, and so forth.

Recently, the variational iteration method introduced by He [1] has been the subject of numerous studies. This paper applies the variational iteration method to two-point boundary value problems. The method was first proposed in 1998 to solve nonlinear equations with fractional derivatives [2]. Then, the method has been applied to delay differential equations [3], Duffing equation with nonlinearity of fifth-order and mathematical pendulum [1], 
generalized KdV and coupled Schrodinger-KdV [4], and some other problems including solitary and compacton-like solutions of nonlinear wave equations, construction of some compact and noncompact structures of Klein-Gordon equations, and different types of nonlinear differential equations [5-19].

The numerical solution of Burger equation is very important because of the equations' application in the approximate theory of flow through a shock wave traveling in a viscous fluid and in the Burger's turbulence model [20]. Some specific one-dimensional Burger equations as an initial value problem rather than a boundary value problem have been solved using VIM $[20,21]$ and modified VIM $[22,23]$. However, these studies have not focused on the effect of nonlinearity in the solution process of VIM.

In this study, VIM solution of nonlinear two-point boundary value problems is considered to focus on the effect of nonlinearity in the convergence behavior of VIM. As a nonlinear two-point boundary value problem, steady Burger equation is considered with changing weights of nonlinearity for the purpose of generalizing and predicting the behavior of nonlinear term in the equation. Five different cases have been solved by using both VIM and FEM, and comparisons are made with respect to the number of iterations and to the error norms based on FEM solutions of the equations with very fine meshes.

\section{Variational iteration method}

According to the variational iteration method, the following differential equation may be considered:

$$
L u+N u=g(x),
$$

where $L$ is a linear operator, $N$ is a nonlinear operator, and $g(x)$ is an inhomogeneous term. Based on the variational iteration method, a correct functional can be constructed as follows:

$$
u_{n+1}(x)=u_{n}(x)+\int_{0}^{x} \lambda\left\{L u_{n}(\tau)+N \tilde{u}_{n}(\tau)-g(\tau)\right\} d \tau,
$$

where $\lambda$ is a general Lagrangian multiplier, which can be identified optimally via the variational theory, the subscript $n$ denotes the $n$ th-order approximation; the second term on the right is called the correction in which $\tilde{u}$ is considered as a restricted variation, that is, $\delta \widetilde{u}=0$. Equation (2.2) is called correction functional. For further details He's studies $[1,2,17,18]$ can be visited.

\section{Finite element method}

The equation to be solved in this study is a nonlinear two-point boundary value problem namely steady Burger equation given as follows:

$$
u^{\prime \prime}-\alpha u u^{\prime}=0, \quad u(0)=0, \quad u(1)=1, \quad x \in[0,1] .
$$

This equation can be linearized using classical linearization technique. To this aim, value of $u$ at any node in an element will be assumed to be known. Modified equation to be solved becomes:

$$
u^{\prime \prime}-\alpha \bar{u} u^{\prime}=0,
$$

where $\bar{u}$ represents the previously known values of $u$. 


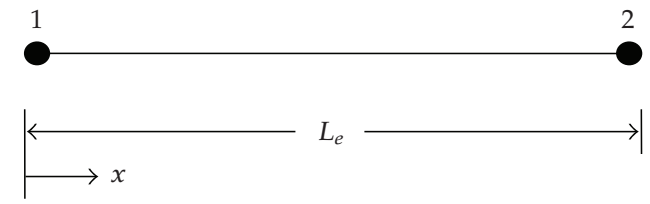

Figure 1: Linear element used in finite element formulation.

Weak form of (3.2) is

$$
(\nabla u, \nabla \psi)-\alpha(\bar{u} \nabla u, \psi)=0,
$$

where $\bar{u}$ is assumed as changing linearly in a two-noded linear element shown in Figure 1. For such an element, corresponding shape functions are defined by the following equations:

$$
\begin{aligned}
& \psi_{1}(x)=1-\frac{x}{L_{e}}, \\
& \psi_{2}(x)=\frac{x}{L_{e}},
\end{aligned}
$$

where $L_{e}$ is the element length. Weak formulation of the equation gives the following coefficient matrices for each element:

$$
\begin{aligned}
& {[K]=\frac{1}{L_{e}}\left[\begin{array}{cc}
1 & -1 \\
-1 & 1
\end{array}\right],} \\
& {\left[L^{*}\right]=\frac{\alpha}{6}\left[\begin{array}{cccc}
-2 \bar{u}_{1} & -\bar{u}_{2} & 2 \bar{u}_{1} & +\bar{u}_{2} \\
-\bar{u}_{1} & -2 \bar{u}_{2} & \bar{u}_{1} & +2 \bar{u}_{2}
\end{array}\right] .}
\end{aligned}
$$

In (3.6), $\bar{u}_{1}$ and $\bar{u}_{2}$ are assumed values of $u$ at the nodes 1 and 2 shown in Figure 1 . Final form of the finite element formulation resulting from classical linearization technique is

$$
\left([K]+\left[L^{*}\right]\right)\{u\}=\{F\} .
$$

The analysis procedure is iterative. First a starting value for $u$ is assumed at the nodes of FEM mesh for the whole domain, and the matrix $\left[L^{*}\right]$ is determined using (3.6). Then (3.7) is solved for $u$. The last step in a typical iteration is the comparison of new values with the previous ones. This comparison is based on an error criterion for the whole domain. The error criterion used in this study is

$$
\sqrt{\left(\frac{\sum_{i=1}^{N}\left|u_{i}^{r+1}-u_{i}^{r}\right|^{2}}{\sum_{i=1}^{N}\left|u_{i}^{r+1}\right|^{2}}\right)}<\varepsilon,
$$

where $N$ is the total number of unknowns in the FEM mesh, $r$ is the iteration number, and $\varepsilon$ is the preselected error tolerance.

If error tolerance obtained from (3.8) is less than preselected error, iteration stops and the results produced by final iteration become the solution from FEM analysis. However, if error tolerance is greater than preselected error, then the assumed value of $u$ (i.e., $\bar{u})$ ) is changed with the new values of current iteration, and the process goes in the same manner until preselected error tolerance is reached. 


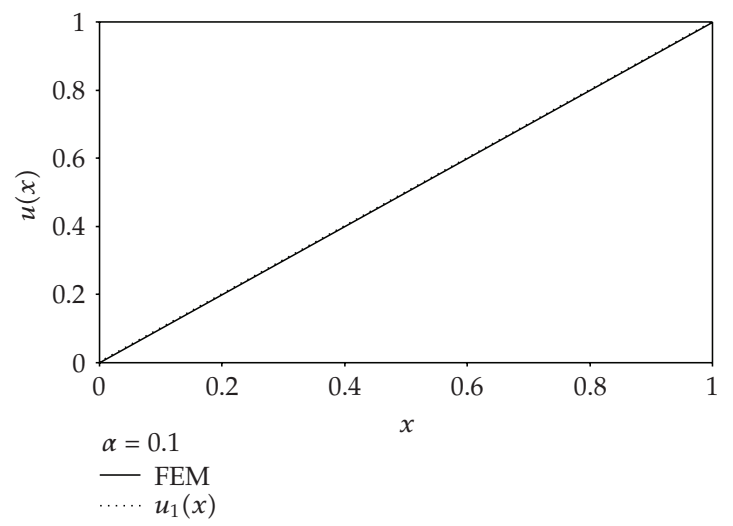

Figure 2: Consecutive VIM approximations compared to FEM solution for $\alpha=0.1$.

100 linear elements are used in the finite element analysis of the problem, and error tolerance is chosen as $10^{-5}$, that is, $\varepsilon=10^{-5}$, for the iteration process. The results obtained from FEM analysis can be seen between Figures 2-6 with the VIM solutions of the same equation.

\section{VIM applications}

We recall steady Burger equation in (3.1) to be solved by using VIM:

$$
u^{\prime \prime}-\alpha u u^{\prime}=0, \quad u(0)=0, \quad u(1)=1, \quad x \in[0,1] .
$$

A correct functional can be derived using VIM as follows:

$$
u_{n+1}(x)=u_{n}(x)+\int_{0}^{x} \lambda\left\{u_{n}^{\prime \prime}(\xi)-\alpha \tilde{u}_{n}(\xi) \tilde{u}_{n}^{\prime}(\xi)\right\} d \xi,
$$

where $\mathcal{\lambda}$ is the Lagrange multiplier and can be easily found as

$$
\lambda=\xi-x .
$$

Hence, the following iteration formula would be obtained:

$$
u_{n+1}(x)=u_{n}(x)+\int_{0}^{x}(\xi-x)\left\{u_{n}^{\prime \prime}(\xi)-\alpha u_{n}(\xi) u_{n}^{\prime}(\xi)\right\} d \xi
$$

Iteration process can be started using initial guess as

$$
u_{0}(x)=A x+B,
$$

where $A$ and $B$ are constants to be determined.

For the analysis, different $\alpha$ values will be considered based on this formulation. $\alpha$ takes values between 0.1 and 100 to represent the increasing power of nonlinearity in the equation. 


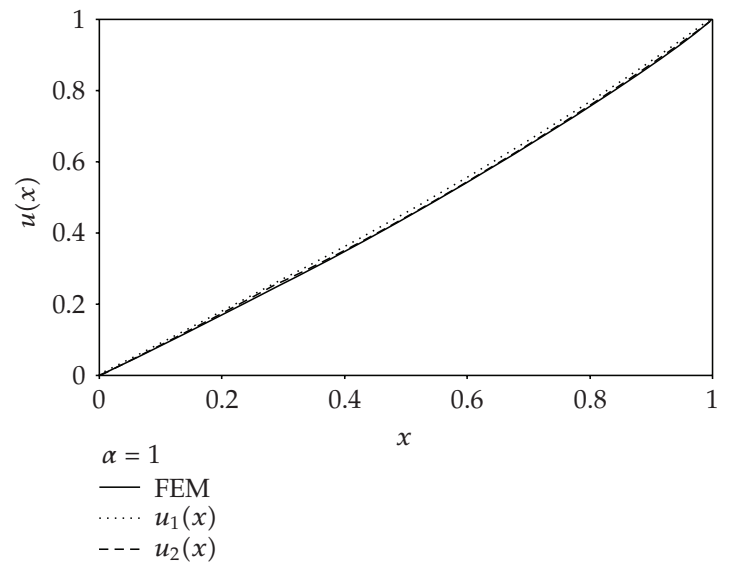

Figure 3: Consecutive VIM approximations compared to FEM solution for $\alpha=1$.

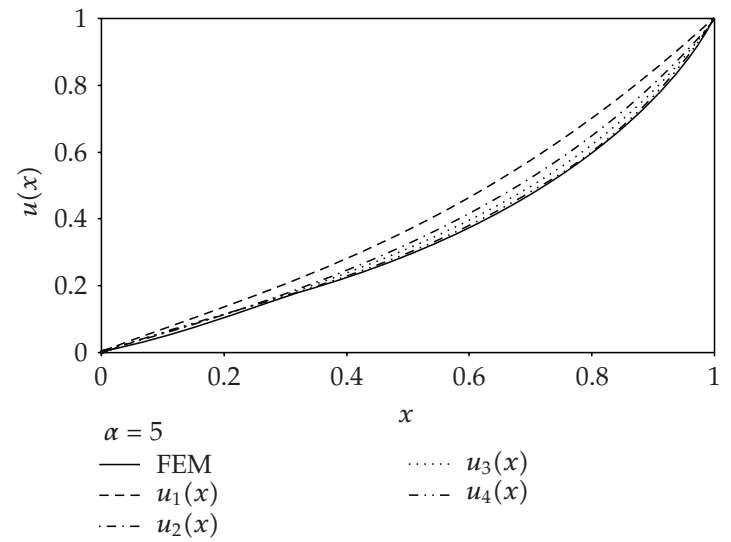

Figure 4: Consecutive VIM approximations compared to FEM solution for $\alpha=5$.

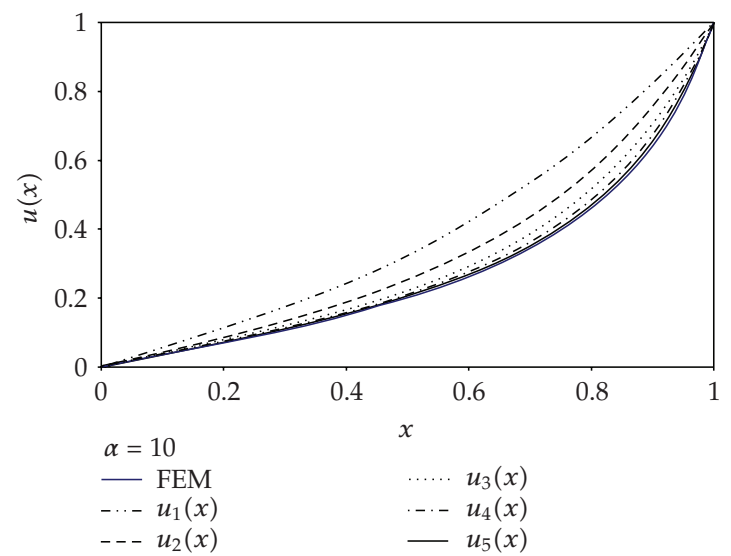

Figure 5: Consecutive VIM approximations compared to FEM solution for $\alpha=10$. 


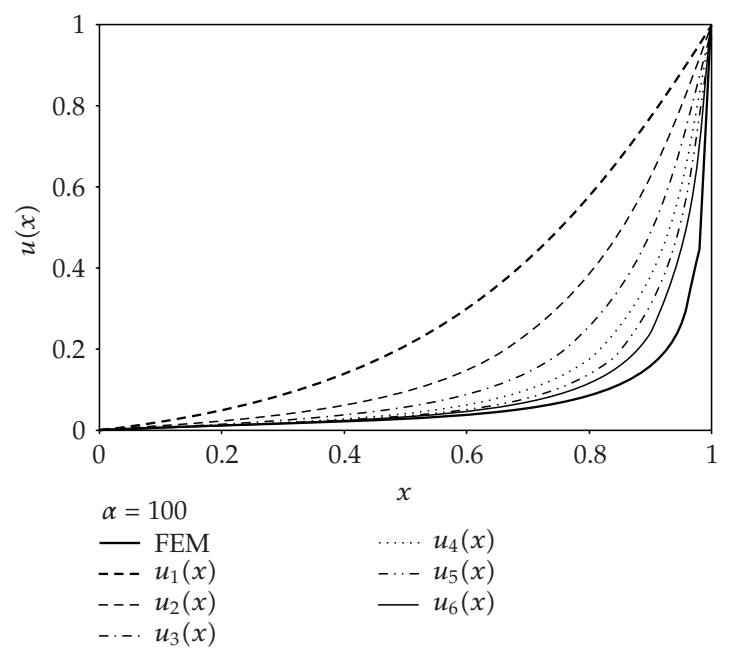

Figure 6: Consecutive VIM approximations compared to FEM solution for $\alpha=100$.

In each case, an error norm is calculated for each iteration to represent the convergence behavior of the solution. The error norm is defined as follows:

$$
\text { Error }=\sqrt{\left(\frac{\sum_{i=1}^{N}\left|u_{\mathrm{FEM}}-u_{\mathrm{VIM}}^{r}\right|^{2}}{\sum_{i=1}^{N}\left|u_{\mathrm{FEM}}\right|^{2}}\right)}
$$

where $N$ is the number of nodes in the FEM mesh, $r$ is the iteration number of the VIM, $u_{\mathrm{FEM}}$ is the FEM solution, and $u_{\mathrm{VIM}}^{r}$ is the VIM solution at the rth iteration.

Case $1(\alpha=0.1)$. The iteration formula for this case is

$$
u_{n+1}(x)=u_{n}(x)+\int_{0}^{x}(\xi-x)\left\{u_{n}^{\prime \prime}(\xi)-(0.1) u_{n}(\xi) u_{n}^{\prime}(\xi)\right\} d \xi
$$

The first iteration gives

$$
u_{1}(x)=0.983867 x+0.0161332 x^{3}
$$

This approximation is very close to the FEM solution of the equation as shown in Figure 2. Error norm defined in (4.6) is calculated as 0.000202871. VIM solution is in perfect agreement with FEM solution even in the first iteration due to the fact that there is considerably weak nonlinearity in the equation. 
Case $2(\alpha=1)$. The iteration formula for this case is

$$
u_{n+1}(x)=u_{n}(x)+\int_{0}^{x}(\xi-x)\left\{u_{n}^{\prime \prime}(\xi)-u_{n}(\xi) u_{n}^{\prime}(\xi)\right\} d \xi
$$

Iterations are

$$
\begin{aligned}
& u_{1}(x)=0.872983 x+0.127017 x^{3} \\
& u_{2}(x)=0.85593 x+0.122103 x^{3}+0.0209023 x^{5}+0.00106493 x^{7}
\end{aligned}
$$

In this case, the number of iterations increased with the increasing nonlinearity effect. Second approximation is in perfect agreement with the FEM solution as shown in Figure 3, and error norms for this case are given in Table 1.

Case $3(\alpha=5)$. The iteration formula for this case is

$$
u_{n+1}(x)=u_{n}(x)+\int_{0}^{x}(\xi-x)\left\{u_{n}^{\prime \prime}(\xi)-(5) u_{n}(\xi) u_{n}^{\prime}(\xi)\right\} d \xi
$$

Iterations are

$$
\begin{aligned}
u_{1}(x)= & 0.649 x+0.351 x^{3} \\
u_{2}(x)= & 0.562735 x+0.263893 x^{3}+0.148501 x^{5}+0.0248711 x^{7}, \\
u_{3}(x)= & 0.534962 x+0.238487 x^{3}+0.127581 x^{5}+0.0690635 x^{7} \\
& +0.0229405 x^{9}+0.00590127 x^{11}+0.000996742 x^{13}+0.0000687683 x^{15}, \\
u_{4}(x)= & 0.525756 x+0.230349 x^{3}+0.121108 x^{5}+\cdots+2.88895 * 10^{-10} x^{31} .
\end{aligned}
$$

As shown in Figure 4, fourth approximation is in very good agreement with the FEM solution. It can be expected that fifth or sixth iteration will be in excellent agreement with the FEM solution. Table 1 shows the decreasing error norms with the increasing iterations for this case.

Case $4(\alpha=10)$. The iteration formula for this case is

$$
u_{n+1}(x)=u_{n}(x)+\int_{0}^{x}(\xi-x)\left\{u_{n}^{\prime \prime}(\xi)-(10) u_{n}(\xi) u_{n}^{\prime}(\xi)\right\} d \xi .
$$

Iterations are

$$
\begin{aligned}
u_{1}(x)= & 0.530662 x+0.469338 x^{3} \\
u_{2}(x)= & 0.415184 x+287297 x^{3}+0.238562 x^{5}+0.0589567 x^{7} \\
u_{3}(x)= & 0.373597 x+0.232624 x^{3}+0.173815 x^{5}+0.13142 x^{7} \\
& +0.0609713 x^{9}+0.0219068 x^{11}+0.00516804 x^{13}+0.000498014 x^{15}, \\
u_{4}(x)= & 0.356649 x+0.211997 x^{3}+0.151217 x^{5}+\cdots+1.90326 * 10^{-8} x^{31} \\
u_{5}(x)= & 0.349601 x+0.203702 x^{3}+0.142429 x^{5}+\cdots+1.51797 * 10^{-17} x^{63}
\end{aligned}
$$


Table 1: Error norms for different $\alpha$ values.

\begin{tabular}{lcccccc}
\hline$\alpha$ & $\operatorname{Err}\left(\mathrm{U}_{1}\right)$ & $\operatorname{Err}\left(\mathrm{U}_{2}\right)$ & $\operatorname{Err}\left(\mathrm{U}_{3}\right)$ & $\operatorname{Err}\left(\mathrm{U}_{4}\right)$ & $\operatorname{Err}\left(\mathrm{U}_{5}\right)$ & $\operatorname{Err}\left(\mathrm{U}_{6}\right)$ \\
\hline 0.1 & 0.000202871 & & & & & \\
1 & 0.014606 & 0.002103 & & & & \\
5 & 0.156351 & 0.063345 & 0.023428 & 0.007741 & & \\
10 & 0.340329 & 0.172809 & 0.084690 & 0.039174 & 0.016889 & \\
100 & 1.804181958 & 1.229720168 & 0.867695947 & 0.623310793 & 0.451744131 & 0.328771988 \\
\hline
\end{tabular}

Figure 5 shows that fifth approximation is in good agreement with the FEM solution. It can be expected that two or more iterations are required for being in excellent agreement with the FEM solution. Decreasing error norms with the increasing iterations for this case can be seen in Table 1.

Case $5(\alpha=100)$. The iteration formula for this case is

$$
u_{n+1}(x)=u_{n}(x)+\int_{0}^{x}(\xi-x)\left\{u_{n}^{\prime \prime}(\xi)-(100) u_{n}(\xi) u_{n}^{\prime}(\xi)\right\} d \xi
$$

Iterations are

$$
\begin{aligned}
u_{1}(x)= & 0.216779 x+0.783221 x^{3}, \\
u_{2}(x)= & 0.108295 x+0.195462 x^{3}+0.423349 x^{5}+0.272895 x^{7}, \\
u_{3}(x)= & 0.0759922 x+0.096247 x^{3}+0.14628 x^{5}+0.22497 x^{7} \\
& +0.212303 x^{9}+0.155159 x^{11}+0.0744542 x^{13}+0.0145939 x^{15}, \\
u_{4}(x)= & 0.0625732 x+0.0652569 x^{3}+0.0816667 x^{5}+\cdots+0.0000153413 x^{31}, \\
u_{5}(x)= & 0.0559616 x+0.052195 x^{3}+0.0584182 x^{5}+\cdots+5.24076 * 10^{-12} x^{63}, \\
u_{6}(x)= & 0.0523792 x+0.0457264 x^{3}+0.0479023 x^{5}+\cdots+1.56741 * 10^{-25} x^{127} .
\end{aligned}
$$

In this final case, the equation is highly nonlinear. As it can be seen from Figure 6, sixth approximation is still not enough for being in good agreement with the FEM solution, and it can be expected that many more iterations are required to reach the close proximity of the FEM solution. For the final case, decreasing error norms with the increasing iterations are also included in Table 1.

\section{Discussion of results}

When all the cases are considered consecutively, a pattern emerges from the number of iterations related with the weight of nonlinearity in the equation. It is obvious that there is a strong relation between the increasing number of iterations required to reach an acceptable solution and the effect of the increasing weight of the nonlinearity in the equation. This phenomenon can also be seen in Figure 7. Based on this figure, it can be inferred that $\alpha$ values, higher than the values considered in this study, will produce very large errors even for considerably many iterations. 


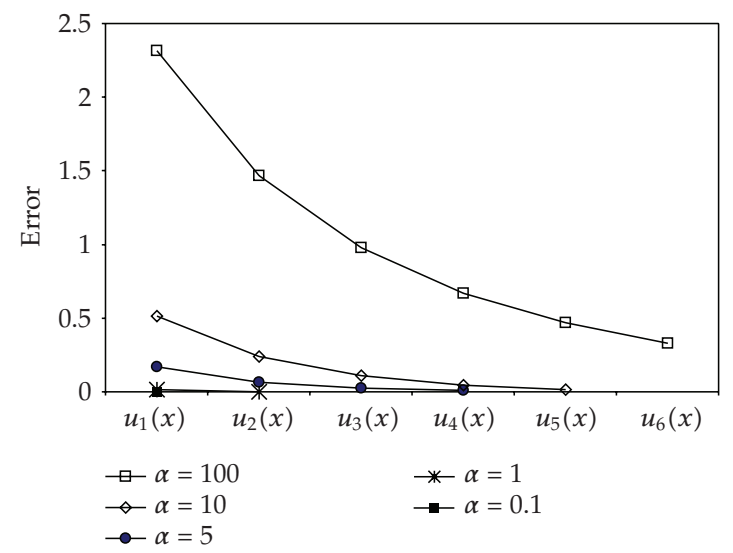

Figure 7: Error estimations of VIM approximations for different $\alpha$ values.

\section{Conclusion}

In this paper, VIM solution of nonlinear two-point boundary value problems with changing nonlinearity effects in the equation is considered. Steady Burger equation is chosen because of its wide range of applications in different disciplines. Five different cases are considered in detail with respect to their nonlinear terms. The results obtained from these cases are compared with the corresponding FEM solutions.

Although FEM is a powerful numerical technique for the solution of differential equations, a fixed mesh with fixed points within the boundary is used for FEM analysis. These fixed points are called "node" in FEM, and the solutions are obtained at the nodes. Important merits of VIM are that it is a kind of analytical solution which does not need a discretized solution domain, and the solution procedure is very simple. The advantage of VIM at this point is that VIM solution is obtained for any arbitrary point instead of fixed points in the domain. Another advantage of VIM is the method which is easy to apply. In addition, application of FEM is much more difficult when compared to VIM in the analysis of nonlinear boundary value problems.

However, as shown from the case studies, when the equation becomes highly nonlinear, the advantages of VIM disappear dramatically compared to FEM. In such a case, many more iterations are required in VIM for an acceptable solution.

As a result, it can be concluded that VIM can be preferred for the analysis of nonlinear two-point boundary value problems when the equation is not dominated by its nonlinear part. For such cases, FEM is much more suitable for the analysis of the problem.

\section{References}

[1] J.-H. He, "Variational iteration method—a kind of non-linear analytical technique: some examples," International Journal of Non-Linear Mechanics, vol. 34, no. 4, pp. 699-708, 1999.

[2] J.-H. He, "Approximate analytical solution for seepage flow with fractional derivatives in porous media," Computer Methods in Applied Mechanics and Engineering, vol. 167, no. 1-2, pp. 57-68, 1998.

[3] J.-H. He, "Variational iteration method for delay differential equations," Communications in Nonlinear Science and Numerical Simulation, vol. 2, no. 4, pp. 235-236, 1997. 
[4] M. A. Abdou and A. A. Soliman, "New applications of variational iteration method," Physica D, vol. 211, no. 1-2, pp. 1-8, 2005.

[5] J.-H. He, "Some asymptotic methods for strongly nonlinear equations," International Journal of Modern Physics B, vol. 20, no. 10, pp. 1141-1199, 2006.

[6] J.-H. He, Non-Perturbative Methods for Strongly Nonlinear Problems, Dissertation.de-Verlag im Internet $\mathrm{GmbH}$, Berlin, Germany, 2006.

[7] J.-H. He, "Variational iteration method for autonomous ODE systems," Applied Mathematics and Computation, vol. 114, no. 2-3, pp. 115-123, 2000.

[8] J.-H. He, "Variational approach to the sixth-order boundary value problems," Applied Mathematics and Computation, vol. 143, no. 2-3, pp. 537-538, 2003.

[9] S. Momani, S. Abuasad, and Z. Odibat, "Variational iteration method for solving nonlinear boundary value problems," Applied Mathematics and Computation, vol. 183, no. 2, pp. 1351-1358, 2006.

[10] J.-H. He and X.-H. Wu, "Construction of solitary solution and compacton-like solution by variational iteration method," Chaos, Solitons E Fractals, vol. 29, no. 1, pp. 108-113, 2006.

[11] E. Yusufoglu, "Variational iteration method for construction of some compact and noncompact structures of Klein-Gordon equations," International Journal of Nonlinear Sciences and Numerical Simulation, vol. 8, no. 2, pp. 153-158, 2007.

[12] H. Tari, D. D. Ganji, and M. Rostamian, "Approximate solutions of K(2,2), KdV and modified KdV equations by variational iteration method, homotopy perturbation method and homotopy analysis method," International Journal of Nonlinear Sciences and Numerical Simulation, vol. 8, no. 2, pp. 203-210, 2007.

[13] Z. M. Odibat and S. Momani, "Application of variational iteration method to nonlinear differential equations of fractional order," International Journal of Nonlinear Sciences and Numerical Simulation, vol. 7, no. 1, pp. 27-34, 2006.

[14] N. Bildik and A. Konuralp, "The use of variational iteration method, differential transform method and adomian decomposition method for solving different types of nonlinear partial differential equations," International Journal of Nonlinear Sciences and Numerical Simulation, vol. 7, no. 1, pp. 65-70, 2006.

[15] J. Biazar and H. Ghazvini, "He's variational iteration method for solving hyperbolic differential equations," International Journal of Nonlinear Sciences and Numerical Simulation, vol. 8, no. 3, pp. 311-314, 2007.

[16] A. Sadighi and D. D. Ganji, "Solution of the generalized nonlinear Boussinesq equation using homotopy perturbation and variational iteration methods," International Journal of Nonlinear Sciences and Numerical Simulation, vol. 8, no. 4, pp. 435-444, 2007.

[17] J.-H. He, "Variational iteration method-some recent results and new interpretations," Journal of Computational and Applied Mathematics, vol. 207, no. 1, pp. 3-17, 2007.

[18] J.-H. He and X.-H. Wu, "Variational iteration method: new development and applications," Computers and Mathematics with Applications, vol. 54, no. 7-8, pp. 881-894, 2007.

[19] S. B. Coşkun and M. T. Atay, "Analysis of convective straight and radial fins with temperaturedependent thermal conductivity using variational iteration method with comparison with respect to finite element analysis," Mathematical Problems in Engineering, vol. 2007, Article ID 42072, 15 pages, 2007.

[20] M. A. Abdou and A. A. Soliman, "Variational iteration method for solving Burger's and coupled Burger's equations," Journal of Computational and Applied Mathematics, vol. 181, no. 2, pp. 245-251, 2005.

[21] M. Moghimi and F. S. A. Hejazi, "Variational iteration method for solving generalized Burger-Fisher and Burger equations," Chaos, Solitons E Fractals, vol. 33, no. 5, pp. 1756-1761, 2007.

[22] T. A. Abassy, M.A. El-Tawil, and H. El Zoheiry, "Toward a modified variational iteration method," Journal of Computational and Applied Mathematics, vol. 207, no. 1, pp. 137-147, 2007.

[23] T. A. Abassy, M. A. El-Tawil, and H. El Zoheiry, "Solving nonlinear partial differential equations using the modified variational iteration Padé technique," Journal of Computational and Applied Mathematics, vol. 207, no. 1, pp. 73-91, 2007. 


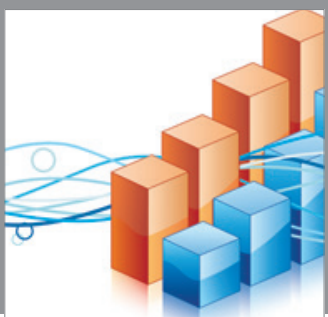

Advances in

Operations Research

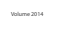

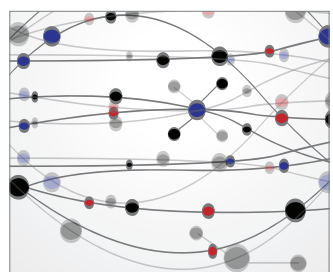

\section{The Scientific} World Journal
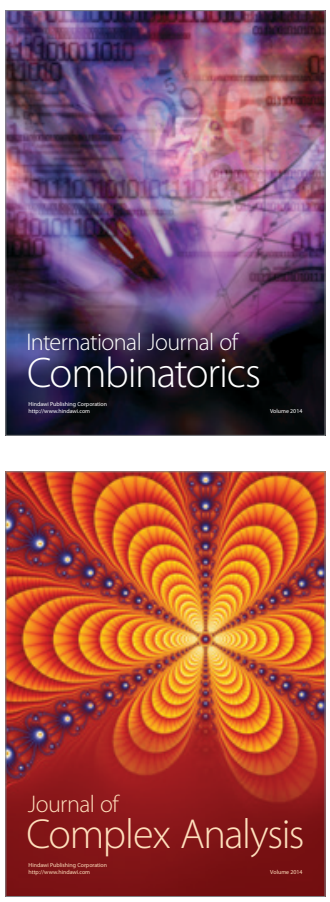

International Journal of

Mathematics and

Mathematical

Sciences
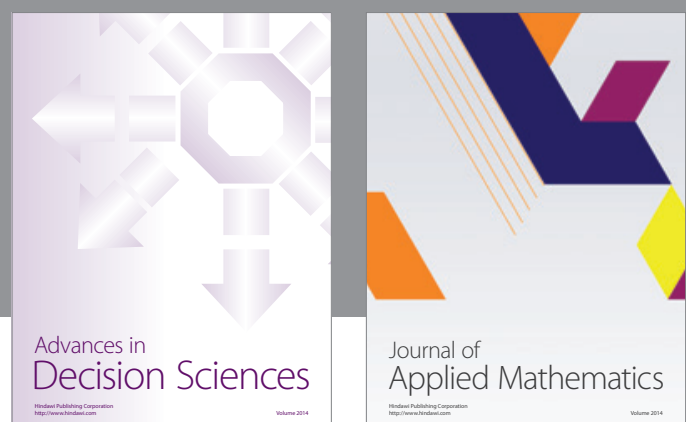

Journal of

Applied Mathematics
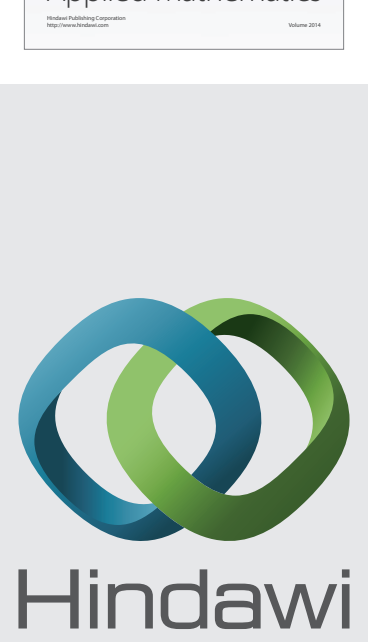

Submit your manuscripts at http://www.hindawi.com
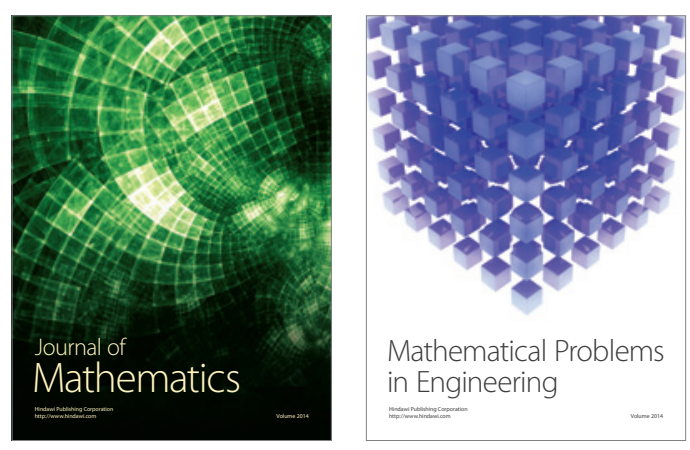

Mathematical Problems in Engineering
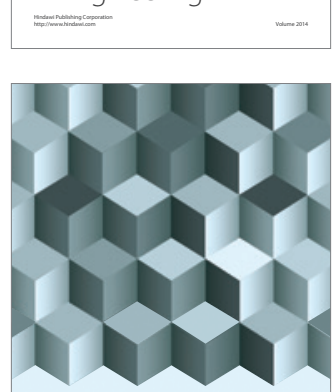

Journal of

Function Spaces
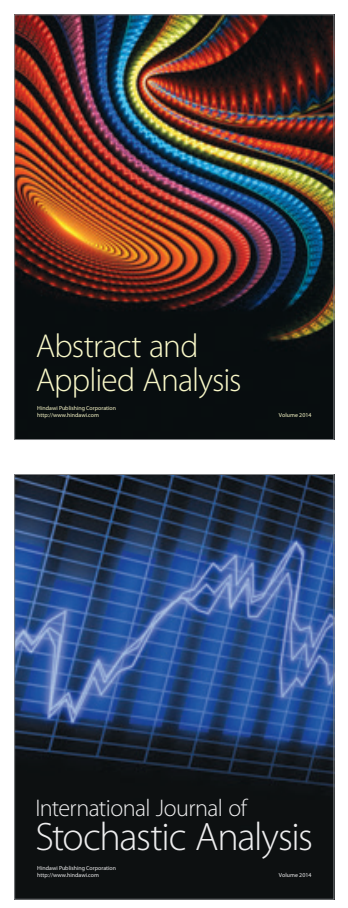

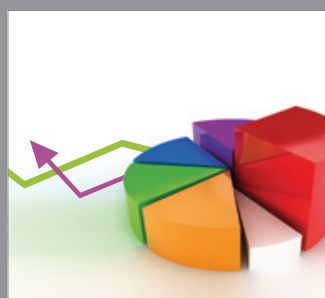

ournal of

Probability and Statistics

Promensencen
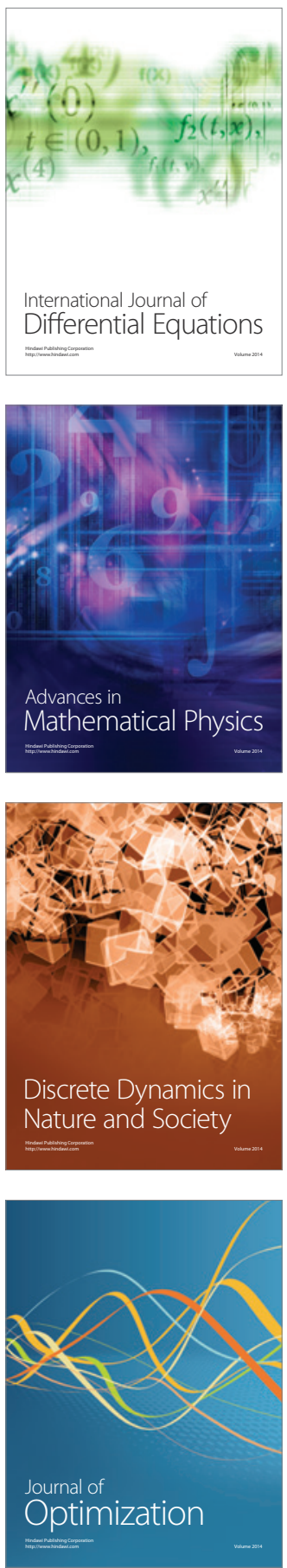